\title{
Unternehmerisches Handeln soll auch im Gesundheitswesen möglich sein
}

\author{
Mit der Spitalfinanzierung über DRG müssen neue Wege für die Abgeltung medi- \\ zintechnischer Innovationen gefunden werden. Melchior Buchs erklärt im Gespräch \\ mit der Schweizerischen Ärztezeitung, weshalb die Medtech-Industrie eine \\ adäquate Finanzierung fordert und wie diese mit dem Selbstverständnis einer \\ wettbewerbsorientierten Branche vereinbar ist.
}

Seit 18 Monaten rechnen die Spitäler mit SwissDRG ab. Wie sieht die Zwischenbilanz aus Ihrer Sicht aus?

Melchior Buchs: Aus der Perspektive der Medizintechnik-Industrie lässt sich feststellen, dass sich das Einkaufsverhalten der Spitäler verändert hat. Ob das nun eine direkte Folge von DRG ist, bleibt dahingestellt. Es kann auch eine Folge allgemeiner Budgetrestriktionen sein. Die Spitäler schenken jedenfalls dem Einkauf vermehrt Aufmerksamkeit. Früher unternahmen sie nicht allzu grosse Anstrengungen, um Kosten einzusparen. Das hat sich geändert. Die Einkaufsverantwortlichen erhalten von den Spitalleitungen klare Sparvorgaben, und es gibt zunehmend Einkaufsgemeinschaften, die gezielter und professioneller arbeiten. Das spüren unsere Mitglieder natürlich.

Die Orthopäden schlugen letzten Sommer Alarm, weil sie billigere statt der geeigneten Implantate einsetzen müssten. Erleben wir wirklich Qualitätsverluste wegen der DRG?

Wir haben Ende März aufgrund dieser Berichte eine Veranstaltung mit den medizinischen Fachgesellschaften im Bereich Chirurgie organisiert. Es zeigte sich, dass sich das so nicht verallgemeinern lässt. Die Fachgesellschaften wollten das nicht so verstanden wissen, dass gute gegen schlechte Qualität ausgetauscht werde. Man wird nun bei den Mitgliedern der FMCH eine Umfrage durchführen, welche Veränderungen sie im Einkauf feststellen, und ob diese qualitative Auswirkungen haben. Wenn SwissDRG richtig und vollumfänglich umgesetzt wird, herrscht mehr Transparenz auf dem Markt, auch für die Patientinnen und Patienten. Und wenn die Transparenz einmal da ist, dann befürchte ich nicht, dass mehr Wettbewerb zu schlechterer Qualität führt. Ein wichtiges Instrument zur Erhöhung der Transparenz ist das Implantat-Register SIRIS, das wir zusammen mit der Schweizerischen Orthopädischen Gesellschaft und anderen Partnern aufgebaut haben. Seit letzten Herbst ist SIRIS nun operativ, und in ein paar Jahren werden die Ergebnisse sichtbar sein.

Muss ich also noch keine Zusatzversicherung für freie Prothesenwahl abschliessen?

Das ist ein interessantes Thema. Natürlich beschäftigen sich die Krankenversicherer damit, was für Produkte sie in nächster Zeit anbieten wollen. Dass es in absehbarer Zeit für Allgemeinversicherte weniger gute Qualität gibt als für Zusatzversicherte, halte ich aber für unwahrscheinlich.

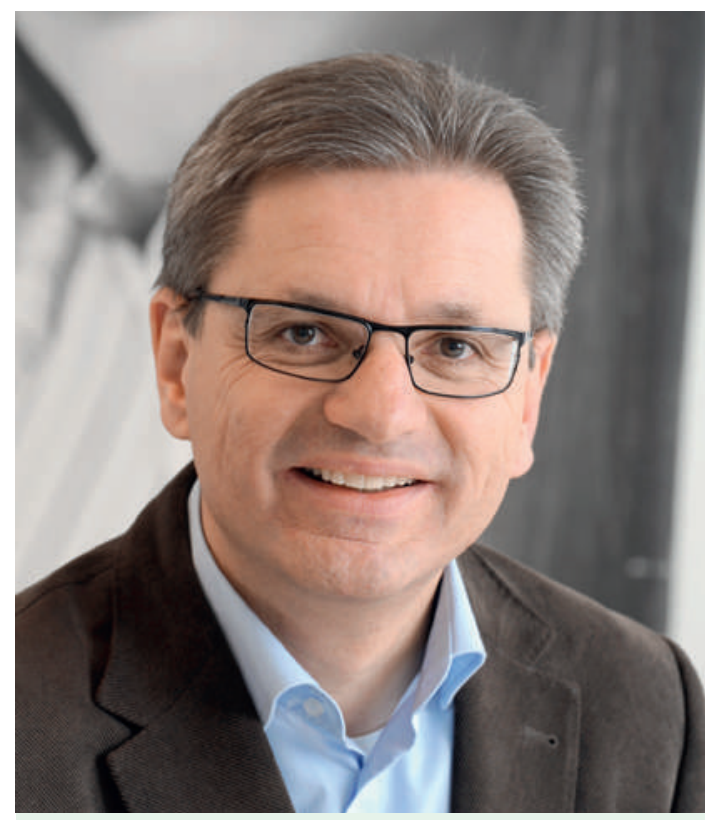

Melchior Buchs, Generalsekretär des Dachverbands der Schweizerischen Handels- und Industrievereinigung der Medizintechnik, FASMED. m.buchs[at] markwalder-partners.ch
Korrespondenz: FASMED-Generalsekretär Worbstrasse 52 CH-3074 Muri/Bern 
FASMED fordert regelmässig wettbewerbsfördernde Rahmenbedingungen. Was verstehen Sie darunter?

Wir wollen, dass selbstverantwortliches, unternehmerisches Handeln auch im Gesundheitswesen möglich ist. Deshalb haben wir uns als Verband nie gegen Öffnungen hin zu mehr Wettbewerb gestellt, auch wenn vielleicht einzelne Mitglieder damit Mühe haben.

\section{«Wir können nicht gleichzeitig für mehr Wettbewerb und gegen Parallelimporte sein.»}

\section{Sind Sie für oder gegen Parallelimporte?}

Wir sprachen uns nicht dagegen aus, als das politisch diskutiert wurde. Wir können nicht gleichzeitig für mehr Wettbewerb und gegen Parallelimporte sein. Die Pharmaindustrie wusste sich gegen Parallelimporte zu schützen, und viele glaubten, dass das auch für die Medizintechnikindustrie gelte. Aber wir wehrten uns explizit nicht. Wir beobachten aber die Entwicklung ganz genau. Die Regeln für die Inverkehrbringung von Medizinprodukten müssen zur Gewährleistung der Patientensicherheit für alle Anbieter die gleichen sein, auch für Reimporteure.

Als das BSV vor zwei Jahren dazu überging, Hörgeräte mittels Pauschalbeiträgen an Hörbehinderte zu finanzieren, wehrten sich die Hersteller gegen diese wettbewerbsfördernde Massnahme. Warum?

Dazu muss ich zuerst sagen, dass die Hörgerätehersteller nicht bei uns organisiert sind. Wir haben die Diskussion im Zusammenhang mit der IVG-Revision aber mit Interesse verfolgt, denn im IVG wird nicht explizit von Hörgeräten gesprochen, sondern von Hilfsmitteln wie zum Beispiel Rollstühlen, und diese sind dann wieder bei uns. Dass sich die Hörgerätehersteller gegen Pauschalbeiträge gewehrt haben, kann ich nicht ganz nachvollziehen. Wichtig ist, dass die freie Wahl des Kunden bestehen bleibt.

Auch im Medizintechnik-Bereich gibt es administrierte Preise.

Die Schweizer Medizintechnik zählt 3700 tätige Unternehmen und gegen 51000 Vollzeitstellen. Sie erzielt einen Umsatz von rund 12,5 Milliarden Franken, was einem Anteil von 2,1\% des BIP entspricht. Sie exportiert Güter im Wert von ca. 11 Milliarden Schweizer Franken. Ihr Dachverband, FASMED, umfasst mit rund 250 Mitgliedsfirmen die bedeutendsten Medtech-Unternehmen aus Industrie und Handel.
Es gibt keine administrierten Preise, sondern in der Mittel- und Gegenständeliste ist ein Höchstvergütungsbetrag (HVB) festgelegt. Darunter ist alles möglich.

Ist es nicht so, dass grundsätzlich der Höchstvergütungsbetrag bezahlt wird?

Die Margen entstehen beim Gross- und Detailhandel. Wenn beispielsweise die Apotheken den HVB in Rechnung stellen, weiss man noch nicht, wie hoch ihre Marge ist und welchen Betrag die Hersteller erhalten haben.

Von hohen Preisen profitiert aber nicht nur der Detailhandel.

Im schweizerischen System, das zum Teil ein Milizsystem ist, erbringen auch Non-Profit-Organisationen wie zum Beispiel die Diabetesgesellschaft Leistungen, die sie über den Verkauf von Produkten zum Teil refinanzieren. Mit der Herabsetzung des HVB für Blutzuckermessgeräte und Teststreifen, die durch den Preisüberwacher gefordert wurde, stellen sich nun aber für die Diabetesgesellschaft existenzielle Probleme. Was die Gesundheitsligen leisten, würde wahrscheinlich im Ausland von staatlichen Stellen erbracht.

Wie hoch sind die Schweizer Preise im Vergleich zum Ausland?

Das ist eine häufig gestellte Frage. Wir wissen natürlich, dass die Preise in der Schweiz höher sind als im Ausland. Der wichtigste Faktor sind sicher die höheren Löhne und auch die Zusatzleistungen, die mit den Produkten verbunden sind. So lange wir aber

\section{«Wir wachsen, doch bei den \\ Renditen sind die Aussichten \\ weniger rosig, denn in der ganzen Welt werden die Gesundheitsbudgets gekürzt.»}

einen freien Markt haben und angesichts des professionelleren Einkaufsverhaltens der Spitäler braucht man kein Prophet zu sein, um vorauszusagen, dass die Preise in der Schweiz sinken werden.

Ein Problem aus Sicht der Medizintechnikbranche ist die Finanzierung von Innovationen unter DRG. FASMED fordert eine Sonderfinanzierung für Innovationen. Weshalb braucht es das?

Mit der Einführung von DRG haben wir bei der Vergütung von neuen Produkten verschiedene Schienen, die parallel, aber zeitlich nicht synchron verlaufen. 


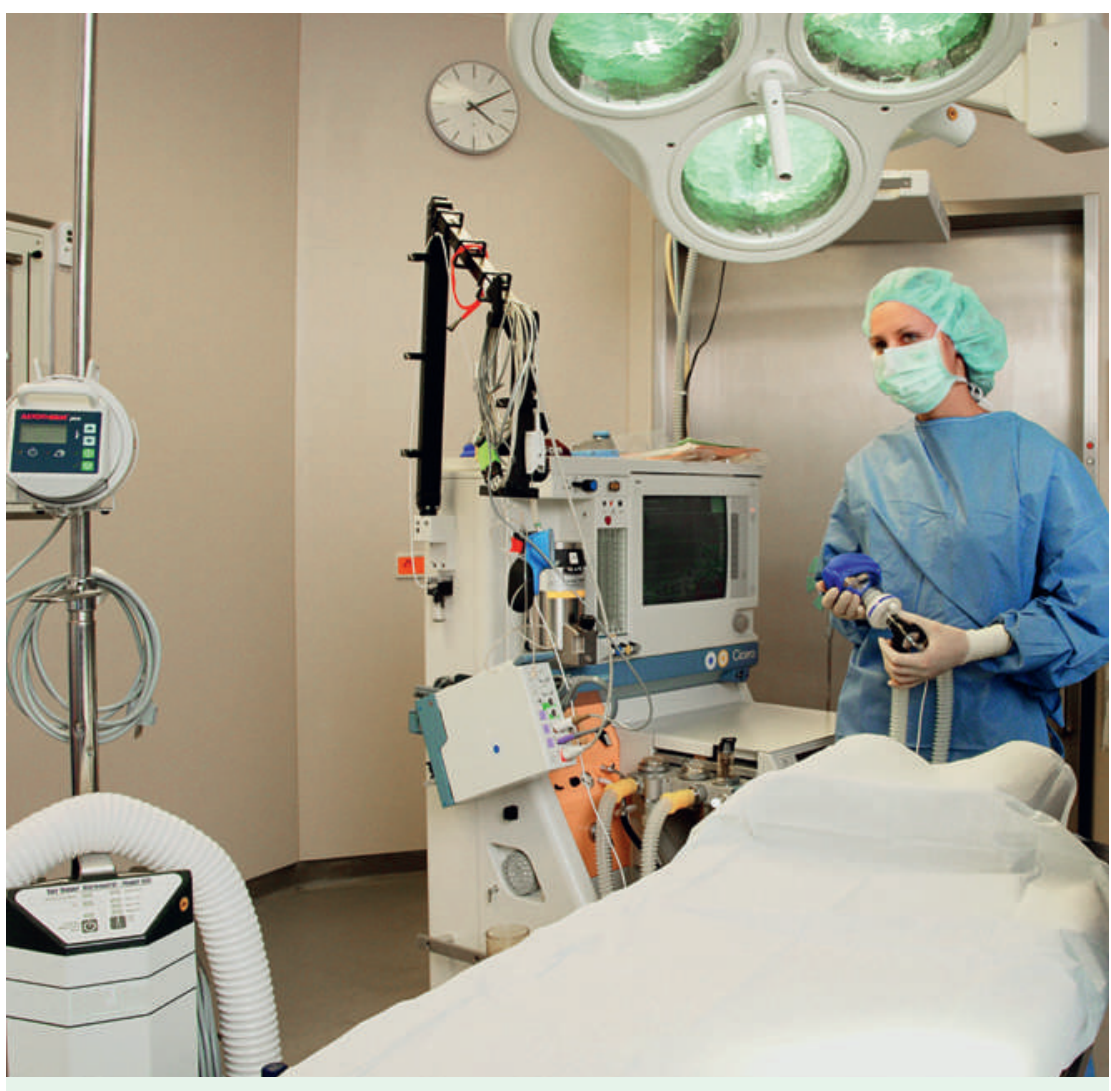

Die Medizinaltechnik ist im ärztlichen Alltag allgegenwärtig.

Wir haben einerseits die Prüfung von Wirksamkeit, Zweckmässigkeit und Wirtschaftlichkeit (WZW) für Leistungen, die von der obligatorischen Grundversicherung vergütet werden. Auf der anderen Seite haben wir den CHOP-Katalog, also die Abbildung einer Leistung im Rahmen der DRG. Ich gebe Ihnen ein aktuelles Beispiel: Mit einem Paclitaxel freisetzenden Ballonkatheter haben wir eine neue medizinische Leistung im Bereich der Koronar-Angioplastie. Hier fand eine WZW-Prüfung statt, die zuständige eidgenössische Kommission für allgemeine Leistungen und Grundsatzfragen empfahl die Leistung zur Ver- gütung, und der Bundesrat nahm sie als vergütungspflichtig in den Anhang 1 der KLV auf. Auf der anderen Seite haben wir noch keinen CHOP-Code, in den diese Leistung integriert ist. Die Spitäler, die diese Innovation anwenden, zahlen also so lange drauf, bis die Leistung in den DRG-Katalog aufgenommen ist. Das dauert aber mehrere Jahre. Für diese Zwischenphase braucht es eine Finanzierung, weil die Vergütungspflicht gegeben ist.

Müssten nicht die Hersteller für diese Zwischenphase aufkommen?

Das Produkt ist zugelassen, es erfüllt also zu diesem Zeitpunkt bereits die WZW-Kriterien. Bis hierher hat die Industrie die Entwicklungskosten getragen. Das Produkt ist auf dem Markt und bräuchte keine zusätzlichen Investitionen. Das Problem ist, dass es im DRGKatalog noch nicht abgebildet und die entsprechende Leistung deshalb während einiger Jahre unterfinanziert ist. Im Moment sind wir daran, dazu mögliche Modelle zu entwickeln. Es gibt Überlegungen über eine Vorfinanzierung durch die Hersteller, aber diese Investition müsste später zurückkommen. In die Entwicklungsstrategie 2013+ der SwissDRG AG wurde diese Frage aufgenommen, und darüber bin ich sehr froh.

Die Wachstumsaussichten der Schweizer Medizintechnikindustrie sind trotz des schwierigen Umfelds nach wie vor gut. Wie zuversichtlich sind Sie, dass es so weitergeht?

Die Nachfrage nach medizinischen Leistungen steigt generell, allein schon aufgrund der demographischen Entwicklung. Wir wachsen, doch bei den Renditen sind die Aussichten weniger rosig, denn in der ganzen Welt werden die Gesundheitsbudgets gekürzt. Da ist es klar, dass sich der Konkurrenzkampf in der Branche zuspitzen wird und folglich auch die Preise unter Druck geraten. Die Firmen, die in der Schweiz produzieren, sind zu 90\% von Exporten abhängig. Aber wir sind innovativ und werden eine Schlüsselbranche für die Schweiz bleiben. 